\title{
OBSERVATION OF SCALING BEHAVIOR IN A COULOMB BLOCKADE SYSTEM
}

\author{
KARSTEN FLENSBERG \\ Dansk Institut for Fundamental Metrologi, \\ Bygn. 307, Anker Engelunds Vej 1 \\ DK-2800 Lyngby, Denmark \\ AND \\ L.W. MOLENKAMP \\ 2. Physikalisches Institut, RWTH Aachen \\ D-52056 Aachen, Germany
}

\begin{abstract}
We describe two experiments to study the influence of fluctuations in the electron charge on the transport properties of a quantum dot. First, we scan a device from single- to double quantum-dot behavior by varying the conductance of a connecting point contact. Second, we measure the dependence of the charging energy on the conductance of the barriers. The experiments are compared with traces obtained from a theory based on a Luttinger liquid type description. This theory predicts a scaling behaviour of the charging energy, in good agreement with our experiments.
\end{abstract}

\section{Introduction}

During the past few years there has been considerable interest in the transport properties of quantum dots[1]. A quantum dot consists of a small metallic island, coupled to external leads by tunnel barriers. At sufficiently low temperatures the energy associated with the addition of one electron to the dot (i.e. the charging energy $U \equiv e^{2} / C$, with $C$ the total dot capacitance) exceeds the thermal energy $\left(k_{\mathrm{B}} T\right)$ and the conductance of the dot is suppressed. This is the Coulomb blockade of the conductance. It can be overcome by adjusting the electrostatic potential of the dot, which is conveniently achieved by varying the voltage $V_{\mathrm{g}}$ of an external gate electrode. At certain values of $V_{\mathrm{g}}$ the electrostatic energy of the dot containing $n$ elec- 
trons becomes equal to that for $(n+1)$ electrons. At these gate voltages transport becomes possible. This results in a periodic series of conductance peaks as a function of $V_{\mathrm{g}}$, known as Coulomb oscillations.

The Coulomb oscillations can only be observed when the electrons are tightly confined to the quantum dot, i.e. when the conductances of the barriers connecting the dot to external leads are small. When the barrier height is decreased, the charge on the island becomes less well-defined due to quantum charge fluctuations and the Coulomb blockade is smeared. This has been studied both experimentally[2] and theoretically[3] in metalic systems which are characterized by a large number of channels connecting the island to the leads. In these systems the Coulomb blockade is gradually reduced as the conductance is increased above the conductance quantum, $2 e^{2} / h$.

For electrostatically defined dots in AlGaAs heterostructures, the contacts to the leads are quantum point contacts with a small number of channels. In this case a fully opened contact has a conductance larger than $2 e^{2} / h$ and the Coulomb blockade is found to disappear[4,5]. This phenomenon has attracted much theoretical attention, since it proved possible to described this behaviour in terms of models know from one-dimensional interacting electron gases $[6,7,8,9]$, so-called Luttinger liquids. The studies of these non-Fermi liquid many electron systems has recently gained new impetus because of its possible relevance to mesoscopic semiconductor structures, both in describing electron tunneling behavior[10,11] and at low magnetic fields[12], as well as in quantum Hall systems where the edge states has been suggested to be describable in terms of Luttinger liquids[13]. An interesting aspect of these suggestions is the possibility of observing effects such as power-law behaviors know from one-dimensional physics in systems which are not truly one-dimensional.

The quantum dot connected to the leads by quantum point contacts may be mapped to a system of coupled one dimensional electron gases, Luttinger liquids $[6,7,8]$, and it can be argued that that the charging energy associated with the addition of an electron to the dot should scale with the conductance of the point-contact barriers, according to $[6,7]$

$$
U^{*} \sim U(1-T)^{N_{c}}
$$

Here, $U$ is the bare charging energy and $U^{*}$ is the effective (or "renormalized") charging energy observed for finite barrier conductance, $N_{c}$ the total number of quantum point contacts leading to the dot, and $T$ the transmission probability of each contact. Here the transmission coefficients are assumed to be equal. For a situation with different $T$ 's the scaling will be governed by the larger of the transmission coefficients. 
In this paper we describe two experiments on a double quantum dot device that were designed to verify the validity of Ref. 1 . First, we study the transition from single- to double-dot behaviour as a function of the transmission of the barrier seperating the two dots. Second, in the double dot regime, we use one dot as an electrometer that measures the charging energy of the other dot. Both experiments can be well modeled by using a standard rate equation approach for the coupled dot system, but with the important addition that we allow the effective energy gap to be modified according to Eq. (1). Part of this research was previously reported by us in ref. [14]. Here we enlarge the discussion and also present further experimental evidence for the interpretation in terms of the scaling law of Eq. (1). Recently, also Waugh et al.[15] reported an experiment which has been shown to be reflect one-dimensional physics[16, 17].

The paper is organized as follows. In Sec. 2 we describe the experimental realization and the operation principle of the coupled dot system, in Sec. 3 the theoretical model is derived and the experimental results and the comparisons with the model are given in Secs. 4 and 3.3.2.

\section{Experimental setup and the electrometer operation}

The devices used in this study are fabricated from a (Al,Ga)As modulation doped heterostructure that contains a two-dimensional electron gas (2DEG) of mobility $2 \times 10^{6} \mathrm{~cm}^{2} \mathrm{~V}^{-1} \mathrm{~s}^{-1}$ and sheet carrier concentration $1.8 \times 10^{11}$ $\mathrm{cm}^{-2}$. The nanostructures are defined electrostatically by split gates, structured using both optical and electron-beam lithography. A schematic layout of the device is shown in Fig. 1. The diameter of each dot is about $1 \mu \mathrm{m}$. All experiments are performed in the mixing chamber of a dilution refrigerator with a minimum base temperature of $20 \mathrm{mK}$ (we estimate the eletron gas temperature to be $150 \mathrm{mK}$ ).

The device is designed to give a very large control over the device parameters since the conductance of all contacts can be adjusted. This means that conductance traces of the quantum dot can be taken for different values of the conductances of the relevant point contacts barriers. Inevitably, there is some cross-talk between different gates which makes the determination of the contact resistances uncertain. We have included this effect in the error estimate of the first experiment. For the second experiment where we study the transition from one to two dots, the cross-talk give rise to an off-set that we determine by fitting to our model. In the experimental results that will be presented, we have measured the conductance $G \equiv G_{14}$ between ohmic contacts 1 and 4, using standard low-frequency lock-in techniques.

Our device can be operated as a semiconductor analogue of the metallic two-dot electrometer device described by Lafarge et al.[18]. The mode-of- 


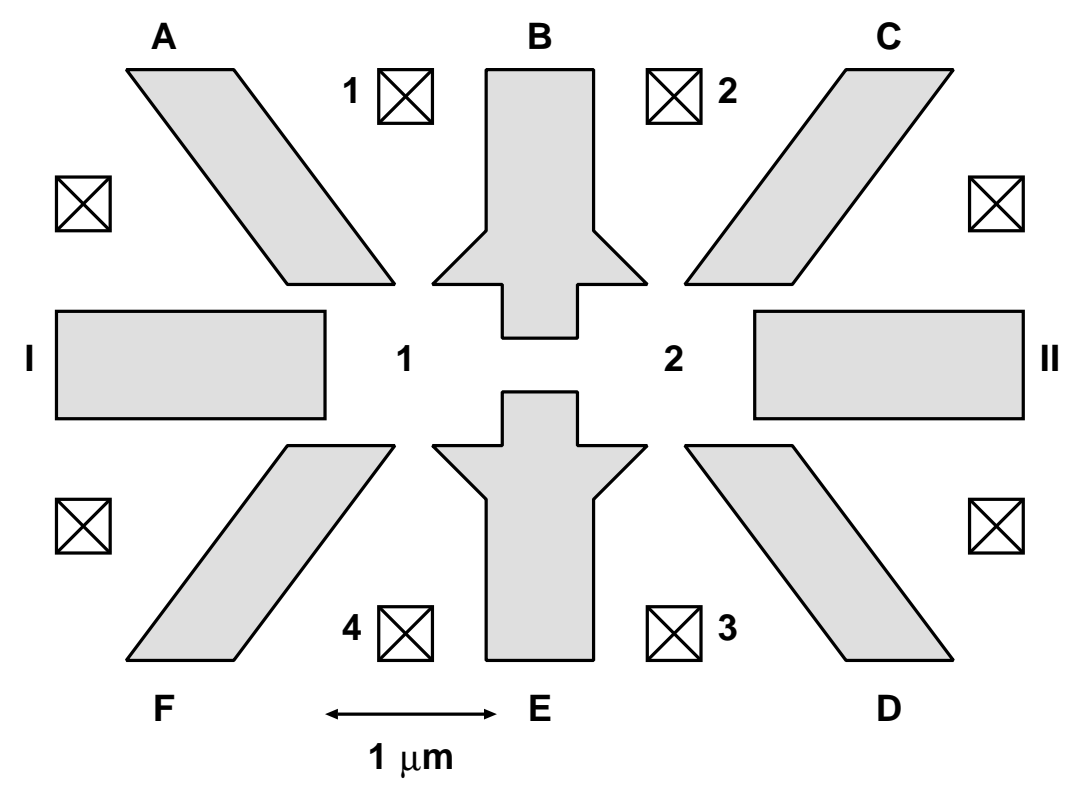

Figure 1. Schematic lay-out of the double dot-sample. The hatched areas denote gates, the crosses ohmic contacts. The lithographic width of one dot is $1 \mu \mathrm{m}$, the distance between two gates defining a point contact is typically $250 \mathrm{~nm}$. We denote the conductance of the contacts connecting to dot 1 by $g_{1}=G_{\mathrm{AB}}$ and $g_{2}=G_{\mathrm{EF}}$, the contacts of dot 2 by $g_{3}=G_{\mathrm{BC}}$ and $g_{3}=G_{\mathrm{ED}}$, the contact connecting the two dots by $g_{5}=G_{\mathrm{EB}}$, and the voltage of the four terminals as $V_{i}, i=1, \ldots, 4$, corresponding to the terminal numbers shown in the figure.

operation of the electrometer experiment is schematically depicted in the bottom panel of Fig. 2. What is measured is the dependence of $G$ on $V_{\mathrm{gII}}$, the voltage on gate II. In our double-dot device, scanning gate II stepwise increases of the occupancy in both dot 1 and dot 2, but with a much shorter stepsize (in $V_{\mathrm{gII}}$ ) in dot 2, because of the larger dot-to-gate capacitance. The trace in Fig. 2 shows the conductance within a $V_{\text {gII-voltage range where } n_{1}}$ only changes by 1 , whereas each superimposed sawtooth corresponds to the addition of one electron to dot 2 . It is then clear that the size of the sawteeth is a measure of the charging energy of dot 2 ; dot 1 thus acts as an electrometer[18] that measures the changes in the potential of dot 2. In the absence of a charging energy of dot 2, the conductance is given by usual Coloumb oscillation peaks, which we have indicated with the dashed line (obtained by setting $U_{2}^{*}=0$ in the equations below). The full curve in Fig. 2 is, in fact, a fit to an experimental trace (dotted curve), using a theory we will discuss below.

Subtraction of the dashed and the full line gives the trace shown in the top panel of the figure. We have also subtracted the same background 


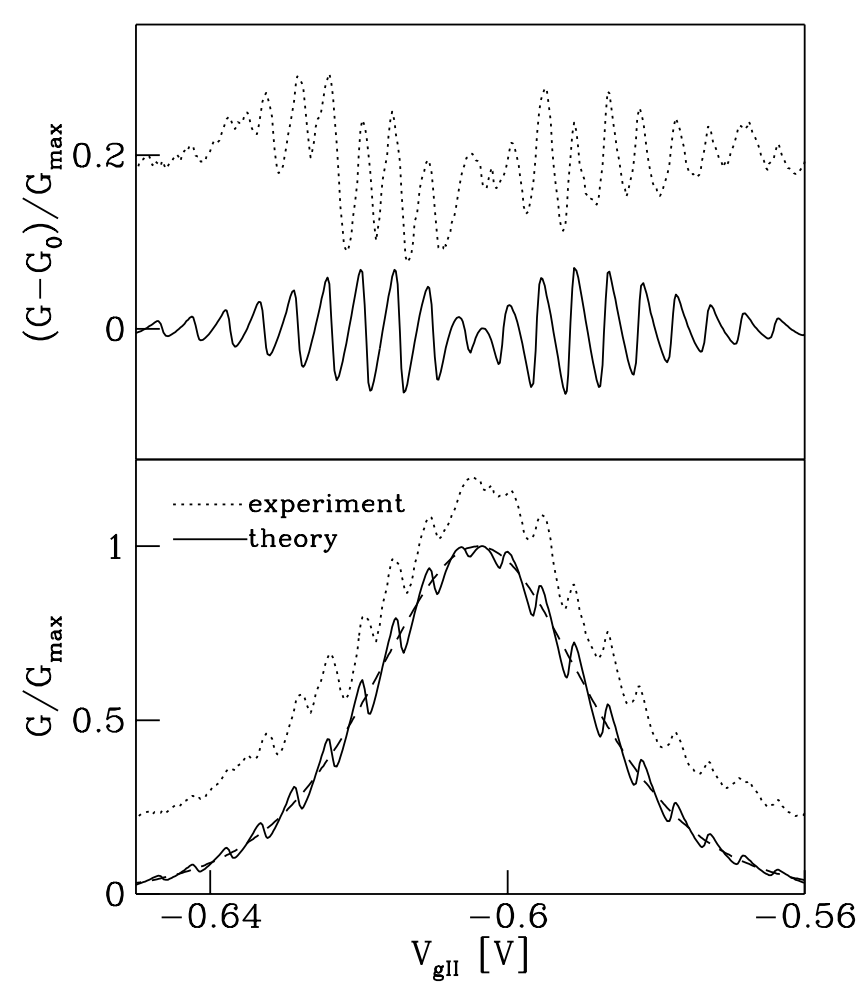

Figure 2. The operating principle of the electrometer experiment: scanning $V_{\text {gII }}$ leads to an increase in $n_{2}$, the number of electrons on dot 2 , which gives sawtooth oscillations in the potential on dot 1 and hence to the conductance through dot 1 . Full lines are results of the model calculation and the bottom panel shows a fit to the experimental trace (dotted curve). The dashed line corresponds to the case where there is no charging energy for the second dot. In the middle panel we have subtracted the conductance of the first dot from the result obtained when there is no charging energy of the second dot. The size of the resulting oscillations thus measures the effective charging energy of dot 2 , which is used for determining $U_{2}^{*}$, as discussed below.

from the experimental trace. Using the subtracted trace, we are able to fit the experimental curves and this fitting procedure is used to determine an effective charging energy for dot in Sec. 4.1.

\section{Setting up the model}

In this section, we describe the theoretical modelling that we use. We apply the standard electrostatic model for the interaction energy and use a rate equation approach to solve for the conductance of the device. Then we incorporate the charge fluctuations in our model by introducing a renor- 
malized charging energy.

\subsection{THE ELECTROSTATIC ENERGY}

The electrostatic energy of our double dot system can be found by considering the various capacitances involved. We define $C_{i}$ as the capacitance between dot $i$ and the leads, $C_{12}$ as the capacitance between the two dots, $C_{g}$ describes the capacitance between a dot and its nearest gate, while $C_{g}^{\prime}$ is the capacitance to the opposite gate. The device is assumed to be symmetric so that $C_{g 1}=C_{g 2}$ and $C_{g 1}^{\prime}=C_{g 2}^{\prime}$. Following, e.g., Ruzin et al.[19] the electrostatic energy is then found to be,

$$
\begin{aligned}
E\left(n_{1}, n_{2}\right)= & U_{11} n_{1}^{2}+U_{22} n_{2}^{2}+U_{12} n_{1} n_{2} \\
& +\left(a_{11} e V_{g 1}+a_{12} e V_{g 2}\right) n_{1}+\left(a_{21} e V_{g 1}+a_{22} e V_{g 2}\right) n_{2},
\end{aligned}
$$

where $n_{i}$ is the number of electrons on dot $i$, and where

$$
\begin{aligned}
U_{11}= & \frac{2 C_{2}+C_{g}+C_{g}^{\prime}+C_{12}}{D}, \\
U_{12}= & \frac{C_{12}}{D}, \\
a_{11}= & \frac{C_{g} C_{g}^{\prime}+\left[C_{g}^{\prime}\right]^{2}+C_{12}\left(C_{g}+C_{g}^{\prime}\right)+2 C_{g}^{\prime} C_{2}}{D}, \\
a_{12}= & \frac{C_{g}^{2}+C_{g} C_{g}^{\prime}+C_{g} C_{12}+2 C_{g} C_{2}}{D}, \\
D= & {\left[C_{g}+C_{g}^{\prime}\right]^{2}+2\left(C_{1}+C_{2}\right)\left(C_{g}+C_{g}^{\prime}+C_{12}\right)+4 C_{2} C_{1} } \\
& +2 C_{12}\left(C_{g}+C_{g}^{\prime}\right),
\end{aligned}
$$

and the quantities $U_{22}, a_{22}$, and $a_{21}$ are obtained by interchanging indices accordingly.

From fitting our experimental data (in absence of quantum fluctuations), we obtain

$$
\begin{aligned}
U_{i i} & \approx 0.13 \mathrm{meV} \\
U_{12} & \approx 0.009 \mathrm{meV} \\
a_{i i} & \approx-0.20 \\
a_{12} & \approx-3.12
\end{aligned}
$$

The electrostatic energy is minimized when

$$
\left(\begin{array}{l}
n_{1} \\
n_{2}
\end{array}\right)=\left(\begin{array}{cc}
2 U_{1} & U_{12} \\
U_{12} & 2 U_{2}
\end{array}\right)^{-1} \cdot\left(\begin{array}{ll}
a_{11} & a_{12} \\
a_{21} & a_{22}
\end{array}\right)\left(\begin{array}{c}
V_{g 1} \\
V_{g 2}
\end{array}\right) \equiv\left(\begin{array}{c}
n_{10} \\
n_{20}
\end{array}\right) .
$$


At zero temperature the number of electrons on the dots is therefore given by

$$
\left\langle n_{i}\right\rangle=\operatorname{Int}\left(n_{i 0}\right) .
$$

Denoting the deviation from the number of electrons that minimize the electrostatic energy by $\delta n_{i}=n_{i}-n_{i 0}$, we get

$$
E=U_{1} \delta n_{1}^{2}+U_{2} \delta n_{2}^{2}+U_{12} \delta n_{1} \delta n_{2}+E\left(n_{10}, n_{20}\right) .
$$

\subsection{SOLUTION OF THE RATE EQUATION}

Next we consider the rate equations for the device. The analysis follows that of Kulik and Shehkter[20], Glazman and Shehkter[21], or Beenakker[22]. The layout is shown in Fig. 1 and we denote the conductances and voltages as explained in the figure and the probability of having charge configuration $\left(n_{1}, n_{2}\right)$ in the two dots as $W\left(n_{1}, n_{2}\right)$. We set up the condition for steady state for dot 1 as

$$
\begin{aligned}
& \frac{d W\left(n_{1}, n_{2}\right)}{d t}= \\
& -W\left(n_{1}, n_{2}\right) \sum_{i=1,4} g_{i} \sum_{ \pm} f\left(E\left(n_{1}, n_{2}\right)-E\left(n_{1} \pm 1, n_{2}\right) \pm e V_{1}\right) \\
& -W\left(n_{1}, n_{2}\right) \sum_{i=2,3} g_{i} \sum_{ \pm} f\left(E\left(n_{1}, n_{2}\right)-E\left(n_{1}, n_{2} \pm 1\right) \pm e V_{1}\right) \\
& +\sum_{ \pm} W\left(n_{1} \pm 1, n_{2}\right) \sum_{i=1,4} g_{i} f\left(E\left(n_{1} \pm 1, n_{2}\right)-E\left(n_{1}, n_{2}\right) \mp e V_{i}\right) \\
& +\sum_{ \pm} W\left(n_{1}, n_{2} \pm 1\right) \sum_{i=2,3} g_{i} f\left(E\left(n_{1}, n_{2} \pm 1\right)-E\left(n_{1}, n_{2}\right) \mp e V_{i}\right) \\
& -W\left(n_{1}, n_{2}\right) g_{5}\left[f\left(E\left(n_{1}, n_{2}\right)-E\left(n_{1}+1, n_{2}-1\right)\right)\right. \\
& \left.+f\left(E\left(n_{1}, n_{2}\right)-E\left(n_{1}-1, n_{2}+1\right)\right)\right] \\
& +W\left(n_{1}+1, n_{2}-1\right) g_{5} f\left(E\left(n_{1}+1, n_{2}-1\right)-E\left(n_{1}, n_{2}\right)\right) \\
& +W\left(n_{1}-1, n_{2}+1\right) g_{5} f\left(E\left(n_{1}-1, n_{2}+1\right)-E\left(n_{1}, n_{2}\right)\right)=0 .
\end{aligned}
$$

Here the function $f$ is the is the usual function given by Fermis Golden Rule,

$$
f(E)=\frac{E}{1-e^{-\beta E}} .
$$

In equilibrium the distrubution function $W$ is given by

$$
W_{0}\left(n_{1}, n_{2}\right)=\exp \left(-\beta E\left(n_{1}, n_{2}\right)\right) / \sum_{n_{1}, n_{2}} \exp \left(-\beta E\left(n_{1}, n_{2}\right)\right) .
$$


In order to find the conductances, we expand in powers of $V_{i}$ and we use the following relations

$$
\begin{aligned}
& W_{0}(x)\left[f(E(x)-E(y)+e V)-W_{0}(y) f(E(y)-E(x)-e V)\right] \\
& \approx e V \beta f(E(x)-E(y)) W_{0}(x)=e V f(E(y)-E(x)) W_{0}(y), \\
& W\left(n_{1}, n_{2}\right)=W_{0}\left(n_{1}, n_{2}\right)+W_{1}\left(n_{1}, n_{2}\right),
\end{aligned}
$$

where $W_{1}$ is of order $V_{i}$.

Since we have a special interest in the case when the device is biased such that current is passed only through dot number 1 and where $g_{1}=$ $g_{4}=G_{0}$, we set $V_{3}=V_{4}=0$. Furthermore, we let the bias across dot 1 be symmetric such that $V / 2=V_{1}=-V_{2}$ (any asymmetry can be absorbed in gate voltages). Under these conditions is it straightforward to verify that $W_{1}=0$ is a solution to the steady state criteria above.

We are now in a position to calculate the current by from the equation

$$
\begin{aligned}
I_{1}= & \frac{g_{1}}{e} \sum_{n_{1} n_{2}}\left[W\left(n_{1}, n_{2}\right) f\left(E\left(n_{1}, n_{2}\right)-E\left(n_{1}+1, n_{2}\right)+e V_{1}\right)\right. \\
& \left.-W\left(n_{1}+1, n_{2}\right) f\left(E\left(n_{1}+1, n_{2}\right)-E\left(n_{1}, n_{2}\right)-e V_{1}\right)\right],
\end{aligned}
$$

and in the special case considered here we get

$$
G=\frac{G_{0}}{4 k T} \sum_{n_{1}, n_{2}} W_{0}\left(n_{1}, n_{2}\right) f\left(E\left(n_{1}, n_{2}\right)-E\left(n_{1}-1, n_{2}\right)\right)
$$

\subsection{MODELLING OF QUANTUM CHARGE FLUCTUATIONS}

Next, we address the question how quantum fluctuations modify the transport properties of the dot device. Assuming an electrostatic model for the interaction energy, it is clear from Eq. (6) that the period of the Coulomb oscillations is uneffected by quantum fluctuations. This is because if the Hamiltonian is given by

$$
H=H_{0}+E\left(n_{1}, n_{2}\right),
$$

(here $H_{0}$ is the single electron Hamiltonian) it is not changed when changing $n_{i 0}$ by one apart from adding a constant to the energy and changing the corresponding average number of electrons by one. Therefore, changing the gate voltage such that the average number of electrons in one of the dots is increased by one does not change the transport properties. ${ }^{1}$ Thus,

\footnotetext{
${ }^{1}$ This argument assumes that the density of states and the tunneling matrix elements are constant within the range of the chemical potential that we are considering.
} 
the spacing of the peak structures are determined by the electrostatics of the system, and only the widths and amplitudes of the peaks are altered by an increase of the number fluctuations. We will model this by invoking a renormalized charging energy $\delta E^{*}$ as described in the following two subsections. This renormalized charging energy is then inserted into Eq. (11) and we have a new expression for the conductance which takes the quantum fluctuations into account

$$
G=\frac{G_{0}}{4 k T} \sum_{n_{1}, n_{2}} W_{0}^{*}\left(n_{1}, n_{2}\right) f\left(\delta E^{*}\left(n_{1}, n_{2}\right)-\delta E^{*}\left(n_{1}-1, n_{2}\right)\right),
$$

where

$$
W_{0}^{*}\left(n_{1}, n_{2}\right)=\exp \left(-\beta \delta E^{*}\left(n_{1}, n_{2}\right)\right) / \sum_{n_{1}, n_{2}} \exp \left(-\beta \delta E^{*}\left(n_{1}, n_{2}\right)\right) .
$$

\subsubsection{Fluctuations of the charge on dot 2}

In one of our experiments the charge on dot 2 is allowed to fluctuate (Sec. 4) by lowering the barriers $\mathrm{BC}$ and $\mathrm{DE}$. The resulting increase in variations of $n_{2}$ will be modelled by using a renormalized value for the charging energy that controls the deviation away from the optimum number of electrons on dot 2. We have

$$
\delta E^{*}\left(n_{1}, n_{2}\right)=\left[U_{1}-U_{12}^{2} / U_{2}\right] \delta n_{1}^{2}+U_{2}^{*}\left(\delta n_{2}-\delta n_{1} U_{12} / 2 U_{2}\right)^{2} .
$$

\subsubsection{Fluctuations of the charge difference between the dots}

In a second experiment, we allow for the charge to fluctuate between the two dots by gradually opening contact $g_{5}$ defined between gates B and E. This results in an effective smaller charging energy for the charge difference between the dots. This we now incorporate in our modelling. Writing the bare electrostatic energy in terms of this difference and the total number of electrons on the two dots, $\Delta n=\delta n_{1}-\delta n_{2}$ and $N=n_{1}+n_{2}$, we have that

$$
E(\Delta n, N)=U_{N}\left(N-N_{0}\right)^{2}+U_{\Delta n}\left(\Delta n-\Delta n_{0}\right)^{2}
$$

where $N_{0}=n_{10}+n_{20}, \Delta n_{0}=n_{10}-n_{20}$, and where

$$
\begin{aligned}
U_{N} & =\left(2 U_{1}+U_{12}\right) / 4 \\
U_{\Delta n} & =\left(2 U_{1}-U_{12}\right) / 4
\end{aligned}
$$

Here we have assumed that $U_{1}=U_{2}$, which is a good approximation in our experiment. 


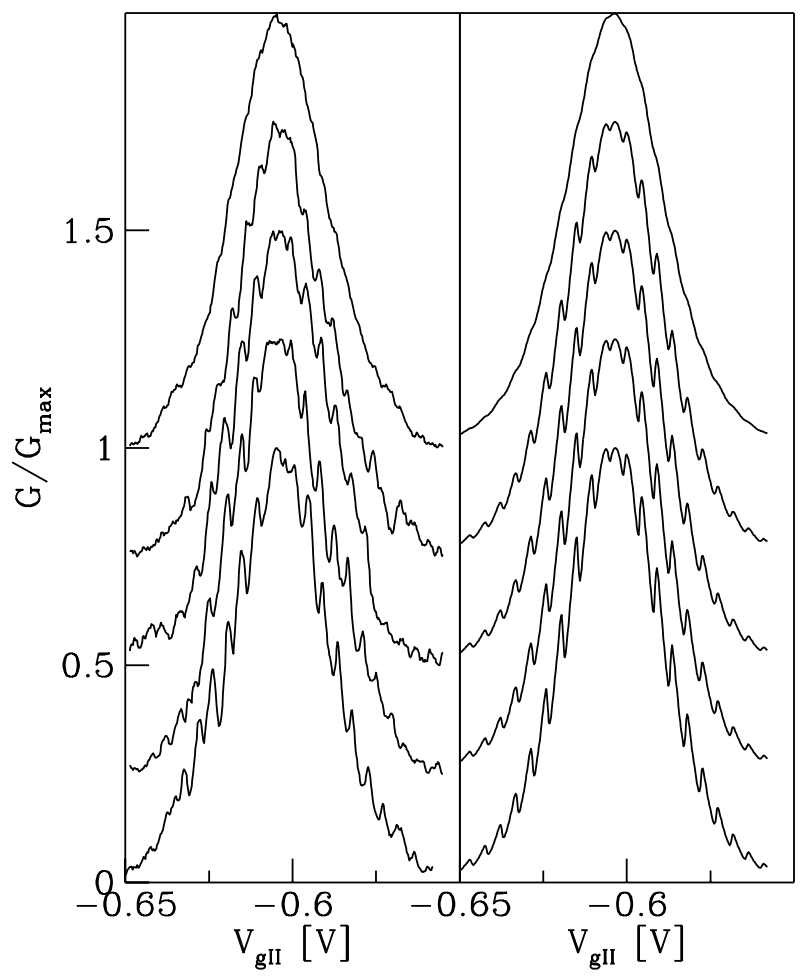

Figure 3. Traces of $G$ versus $V_{\mathrm{gII}}$ in an electrometer experiment, where the conductance of barriers $\mathrm{BC}$ and $\mathrm{DE}$ is varied. An offset of $0.25 \times G / G_{\max }$ is used between consecutive curves. Left panel: Experimental dat a, where from top to bottom $g_{2}, g_{3} \approx 1.3,0.65,0.43$, 0.14 and $0.05 \times e^{2} / h$. In all traces $g_{1}, g_{4}, g_{5} \approx 0.05 \times e^{2} / h$. Right panel: The results of our model calculations.

Now in the case where the charge difference $\Delta n$ exhibit quantum fluctuations it may be modeled by renormalizing the charging energy associated with changes in $\Delta n$. This leads to the model for the renormalized energy

$$
\delta E^{*}(\Delta n, N)=U_{N}\left(N-N_{0}\right)^{2}+U_{\Delta n}^{*}\left(\Delta n-\Delta n_{0}\right)^{2} .
$$

\section{Fluctuations of the charge on $\operatorname{dot} 2, N_{c}=2$}

We will now discuss an experiment that measures the scaling behavior of $U_{2}$ with the conductance of barriers $g_{2}$ and $g_{3}$. In the left panel of Fig. 3 we 
plot $G$ versus $V_{\mathrm{gII}}$ in a series of measurements where the barriers between dot 2 and the wide 2DEG are gradually adjusted from the metallic to the tunneling regime. From top to bottom we have $g_{2}, g_{3} \approx 1.3,0.65,0.43,0.14$ and $0.05 \times e^{2} / h$, respectively, while in all traces $g_{1}, g_{4}, g_{5} \approx 0.05 \times e^{2} / h$, so that dot 1 is always fully in the Coulomb blockade regime. One clearly observes the sawtooth structure on the dot-1 Coulomb oscillation due to the electrometer effect. In addition, one finds that for increasing conductances $g_{2}$ and $g_{3}$ the sawtooth feature is much less pronounced.

The right panel of Fig. 3 shows lineshapes calculated from Eqs. (13), (15) and (1). In order to obtain a consistent set of fits, we first determine the parameters introduced in Sec. $3\left(U_{i}=0.13 \mathrm{meV}, U_{12}=0.009 \mathrm{meV}\right.$, $a_{i i}=-0.20$, and $a_{12}=-3.12$ ) from a fit of Eqs. (11) and (6) to the bottom trace of the left panel of Fig. 3 -, which is the same as the experimental (dotted) trace in the bottom panel of Fig. 2 - where both dots are fully in the tunneling regime. The upper curves are then obtained from Eqs. (13) and (15), keeping the same values for $U_{1}, U_{12}$, and $a_{i j}$ while varying $U_{2}^{*}$ with $g_{2}, g_{3}$ according to Eq. (1) with $N_{c}=2$. This is because there are two contacts that allow tunneling. The results of the model calculation is shown in the right hand panel of Fig. 3 and we see that very nice agreement between theory and experiments is found.

\subsection{DETERMINATION OF THE EFFECTIVE CHARGING ENERGY}

In order to determine the renormalized charging within our model more accurately, we present in this section a fitting procedure for finding $U_{2}^{*}$ according to Eqs. (13) and (15). By subtracting the trace that is expected for a Coulomb island without coupling to a dot 2 (the dashed curve in Fig. 2 ), we obtain the traces shown in Fig. 4. By doing the same for the model calculation we have producted the dashed curves in Fig. 4 by changing $U_{2}^{*}$ only. The optimum values of $U_{2}$ are found by comparing the average heights of the peaks in subtracted curves thus producing a relation between the peak heights and $U_{2}^{*}$. This procedure allows us to determine the scaled charging energy of dot 2 fairly accurately. The results of our fitting procedure including estimates for the uncertaincies are shown in Fig. 5. The data is seen to be well described by the $(1-T)^{2}$ behavior predicted by Eq. (1).

\section{The one-to-two dots experiment, $N_{c}=1$}

Next we describe a second experiment where we study the transition from a single dot to a double dot Coulomb blockade device. This is done by adjusting the barrier BE, that connects the two dots The data presented in the left panel of Fig. 2 are plots of $G\left(\equiv G_{14}\right)$ versus $V_{\text {gII }}$ for various 


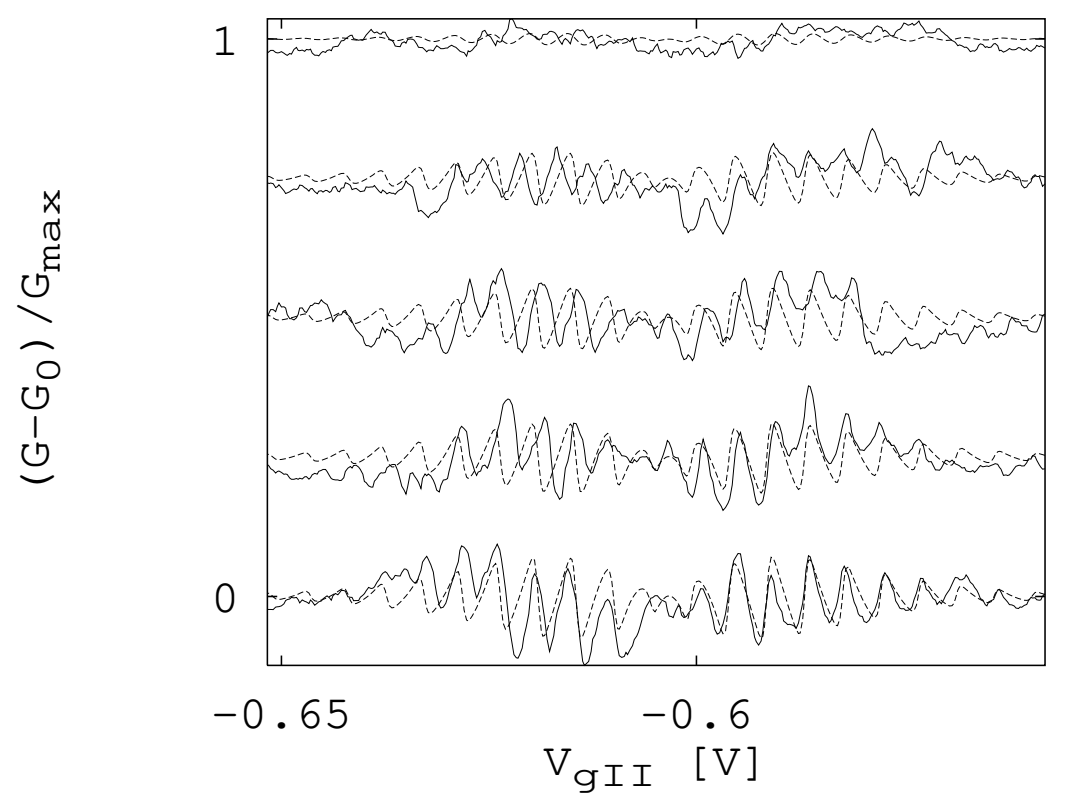

Figure 4. Traces of $G$ versus $V_{\text {gII }}$ for the same data as in Fig. 3 but with the conductance corresponding to no charging of dot 2 subtracted. See also Fig. 2. Curves of this type is used to determine the best fit for the renormalized charging energy $U_{2}^{*}$ and the figure shows the best fit obtained.

adjustments of the conductance $g_{5}$. Barriers $\mathrm{AB}, \mathrm{BC}, \mathrm{DE}$, and $\mathrm{EF}$ are all adjusted well into the tunneling regime, $g_{1}, g_{2}, g_{3}, g_{4} \approx 0.05 \times e^{2} / h$. From top to bottom, $g_{5}$ varies from 0.9 to $0.14 \times e^{2} / h$. Qualitatively one may interpret the data in this figure in a straightforward manner: for $g_{5} \approx 2 e^{2} / h$ the device may be regarded as a single dot. One then expects the rather regular, periodically spaced Coulomb oscillations that are indeed observed in the top trace. On decreasing $g_{5}$, the electrons are confined more and more to either dot 1 or dot 2 . As a result, a beating pattern evolves due to the differences in capacitance between gate II and dots 1 and 2. Finally, for $g_{5} \approx 0.01 \times e^{2} / h$ the device is in the limit where dots 1 and 2 are two separate (but capacitatively coupled) quantum dots.

It is clear that a quantitative discussion of the transition from one to dots must allow for fluctuations in the charge difference $\left(n_{1}-n_{2}\right)$ between 


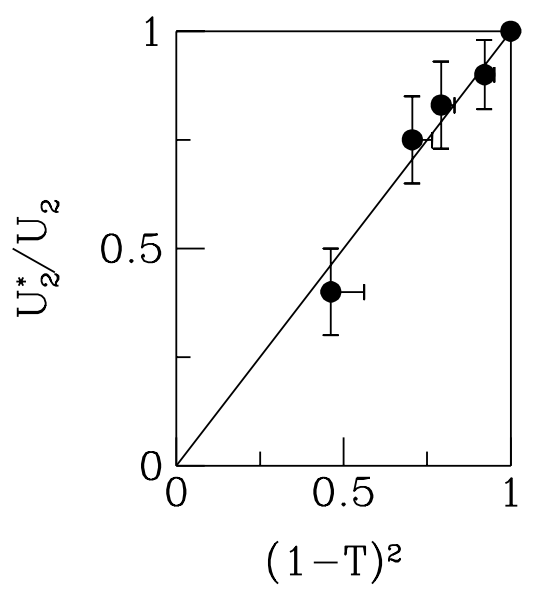

Figure 5. Plot of the ratio $U_{2}^{*} / U_{2}$ between "renormalized" and bare charging energy of dot 2 , versus $(1-T)^{2}$, where $T$ is the transmission of barriers $\mathrm{BC}$ and DE that control the coupling between dot 2 and the external leads. The linear dependence found in this plot support the validity of a scaling law of the type in Eq. (1).

the two dots. Such a model was described above in Sec. 3.3.2. The scaling of the charging energy that controls the charge difference between the two dots, $U_{\Delta n}^{*}$, is assumed to be given by Eq. (1) but now with $N_{c}=1$, since only one contact is connecting the dots. Notice that in the experiment described previously, we had $N_{c}=2$.

The righthand panel of Fig. 6 shows the results of the model calculation using Eqs. (13),(19) and (1). We see that the data is reasonably well reproduced. However, we also note that our calculations display an increase in the width of the dot 1 Coulomb resonance with increasing $g_{5}$, which is not observed experimentally. The reason for this discrepancy is presently unclear.

\section{Discussion and summary}

In conclusion, we have performed and analyzed experiments aimed at understanding the role of charge fluctuations in the transport properties of quantum dots. We find that the dependence of the charging energy of a quantum dot on the conductance of the point contact tunnel barriers can be well described using a scaling equation. We have speculated that this behavior reflects an underlying one-dimensional physics. The point contacts are one-dimensional in the sense that the transmit only one channel. It has been argued that Coulomb blockade systems connected by quantum point contacts can be described in terms of Luttinger liquid type models $[6,7,8]$ and we view the present experimental results as support of this 


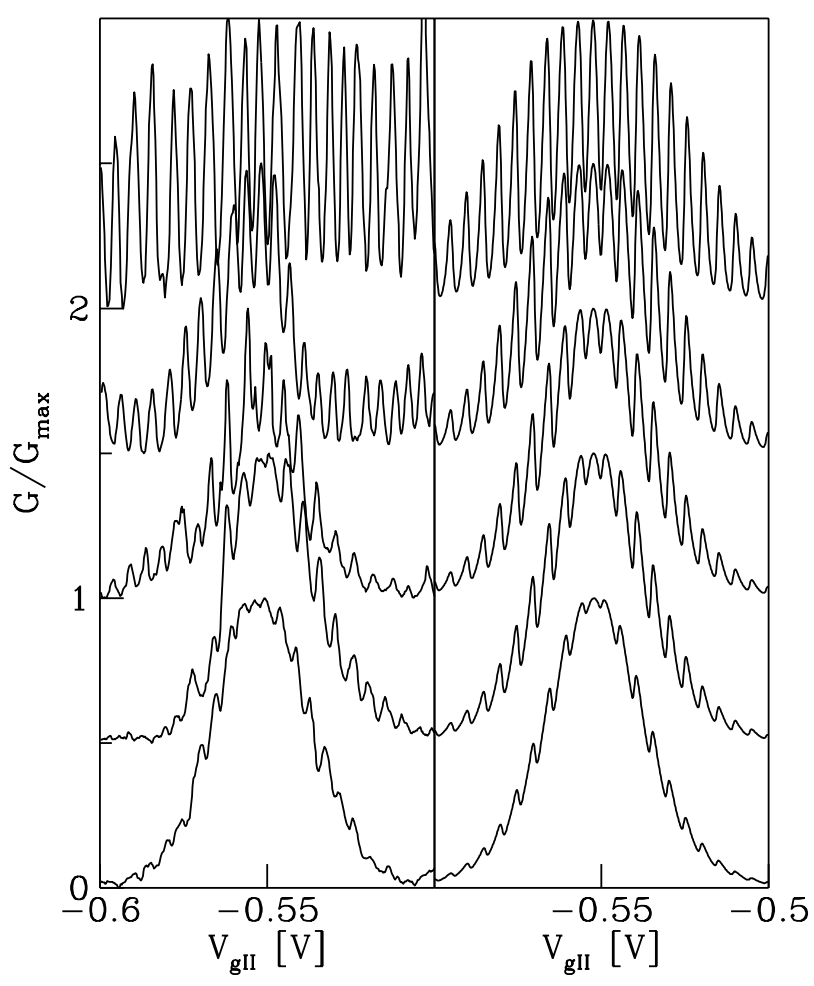

Figure 6. $G$ versus $V_{\text {gII }}$ in an experiment where $g_{5}$ is varied, so that the device changes from a single to a double quantum dot. An offset of $0.5 \times G / G_{\max }$ is used between consecutive curves. Left panel: Experimental traces, where, from top to bottom, $g_{5}=$ $0.9,0.5,0.26$, and $0.14 e^{2} / h$, respectively. During this experiment all other tunnelbarriers are kept at a conductance of about $0.05 e^{2} / h$. Right panel: Theoretical curves using Eq. (19) for $\delta E^{*}$.

scenario. Another recent experiment using a double dot structure[15] has also measured a change of the charging energy as a function of the conductance of a point contact connecting the two dots. The results of this investigation have also been interpreted in terms of a Luttinger liquid type models $[16,17]$. However, in that interpretation a more complete theoretical expression than our Eq. (1) for the case $N_{c}=1$ has been used.

It would be useful to verify the validity of the scaling equation for other power laws, which could be accomplished, e.g., by performing experiments in a high magnetic field, or by varying a different number of tunnelbarriers.

Acknowledgement - We thank M. Kemerink for performing part of the experiments, and O. J. A. Buyk and M. A. A. Mabesoone for expert 
technical assistance. The heterostructures were grown by C. T. Foxon at Philips Research Laboratories in Redhill (Surrey, UK).

\section{References}

1. D. V. Averin and K. K. Likharev, in Mesoscopic Phenomena in solids, edited by B. L.Al'tshuler, P. A. Lee, and R. A. Webb (Elsevier, Amsterdam, 1990).

2. L. J. Geerligs, V. F. Anderegg, and C. A. van der Jeugd, Europhys. Lett. 10, 79 (1989); A. N. Cleland, J. M. Schmidt, and J. Clarke, Phys. Rev. Lett., 64, 1565 (1990).

3. G. Schön and A. D. Zaikin, Phys. Rep. 198, 237 (1990); A. A. Odintsov, Zh. Eksp. Teor. Fiz. 94, 312 (1988) [Sov. Phys. JETP 67, 1265 (1988)]; S. V. Panyukov and A. D. Zaikin, J. Low Temp. Phys. 73, 1 (1988); W. Zwerger and M. Scharpf, Z. Phys. B - Condensed Matter 85, 421 (1991); K. Flensberg and M. Jonson, Phys. Rev. B 43, 7586 (1991); A. D. Zaikin and S. V. Panyukov, Physics Letters A 183, 115 (1993); H. Schöeller and G. Schön, Physica B 203, 423 (1994); G. Falci, J. Heins, G. Schön, and G. T. Zimanyi, Physica B 203, 409 (1994); H. Grabert, Phys. Rev. B 50, 17364 (1994).

4. L. P. Kouwenhoven et al., Z. Phys. B - Condensed Matter 85, 367 (1991).

5. E. B. Foxman et al., Phys. Rev. B 46, 10020 (1993).

6. K. Flensberg, Phys. Rev. B 48, 11156 (1993).

7. K. Flensberg, Physica B 203, 432 (1994).

8. K. A. Matveev, Phys. Rev. B 51, 1743 (1995).

9. A. Furusaki and K. A. Matveev, Phys. Rev. Lett. 75, 709 (1995).

10. C. L. Kane and M. P. A. Fisher, Phys. Rev. Lett. 68, 1220 (1992); Phys. Rev. B 46, 15233 (1992).

11. K. A. Matveev and L. I. Glazman, Phys. Rev. Lett 70, 990 (1993); Physica B 189, 266 (1993).

12. I.L Aleiner and L.I. Glazman, Phys. Rev. B 52, 11296 (1995).

13. X.G. Wen, Phys. Rev. Lett. 64, 2206 (1990); Phys. Rev. B 41, 12838 (1990): ibid 445708 (1991).

14. L. W. Molenkamp, K. Flensberg, and M. Kemerink, Phys. Rev. Lett. 75, 4282 (1995).

15. F. R. Waugh et al., Phys. Rev. Lett. 75, 705 (1995).

16. K. A. Matveev, L. I. Glazman, and H. U. Baranger, cond-mat/9504099.

17. J. M. Golden and B. I. Halperin, cond-mat/9505007.

18. P. Lafarge et al., Z. Phys. B 85, 327 (1991); T. A. Fulton, P.L. Gammel, and L. N. Dunkleberger, Phys. Rev. Lett. 67, 3148 (1991).

19. I. Ruzin, V. Chandrasekhar, E. Levin, and L. Glazman, Phys. Rev. B 45, 13469 (1992).

20. I. Kulik and R. Shehkter, Sov. Phys. JETP 41, 308 (1975).

21. L. I. Glazman and R. Shehkter, J. Phys. Condens. Matter 1, 5811 (1989).

22. C. Beenakker, Phys. Rev. B 44, 1646 (1991). 